\title{
Incretin, insulinotropic and glucose-lowering effects of whey protein pre-load in type 2 diabetes: a randomised clinical trial
}

\author{
Daniela Jakubowicz • Oren Froy • Bo Ahrén • \\ Mona Boaz • Zohar Landau • Yosefa Bar-Dayan • \\ Tali Ganz • Maayan Barnea $\cdot$ Julio Wainstein
}

Received: 11 April 2014 / Accepted: 2 June 2014 /Published online: 10 July 2014

(C) Springer-Verlag Berlin Heidelberg 2014

\begin{abstract}
Aims/hypothesis Since protein ingestion is known to stimulate the secretion of glucagon-like peptide-1 (GLP-1), we hypothesised that enhancing GLP-1 secretion to harness its insulinotropic/beta cell-stimulating activity with whey protein pre-load may have beneficial glucose-lowering effects in type 2 diabetes.

Methods In a randomised, open-label crossover clinical trial, we studied 15 individuals with well-controlled type 2 diabetes who were not taking any medications except for sulfonylurea or metformin. These participants consumed, on two separate days, $50 \mathrm{~g}$ whey in $250 \mathrm{ml}$ water or placebo ( $250 \mathrm{ml}$ water) followed by a standardised high-glycaemic-index breakfast in a hospital setting. Participants were randomised using a coin flip. The primary endpoints of the study were plasma
\end{abstract}

Electronic supplementary material The online version of this article (doi:10.1007/s00125-014-3305-x) contains peer-reviewed but unedited supplementary material, which is available to authorised users.

D. Jakubowicz $(\bowtie) \cdot$ Z. Landau $\cdot$ Y. Bar-Dayan · T. Ganz •

J. Wainstein

Diabetes Unit, E. Wolfson Medical Center, Sackler Faculty of Medicine, Tel Aviv University, 62 Halochamim, 58100 Holon, Israel

e-mail: daniela.jak@gmail.com

O. Froy $(\bowtie) \cdot$ M. Barnea

Institute of Biochemistry, Food Science and Nutrition, The Robert H.

Smith Faculty of Agriculture, Food and Environment, The Hebrew

University of Jerusalem, 1 Herzel, 76100 Rehovot, Israel

e-mail: oren.froy@mail.huji.ac.il

B. Ahrén

Department of Clinical Sciences, Faculty of Medicine, Lund

University, Sölvegatan 19, 22184 Lund, Sweden

M. Boaz

Epidemiology and Research Unit, E. Wolfson Medical Center,

Sackler Faculty of Medicine, Tel Aviv University, 62 Halochamim,

58100 Holon, Israel concentrations of glucose, intact GLP-1 and insulin during the 30 min following meal ingestion.

Results In each group, 15 patients were analysed. The results showed that over the whole $180 \mathrm{~min}$ post-meal period, glucose levels were reduced by $28 \%$ after whey pre-load with a uniform reduction during both early and late phases. Insulin and C-peptide responses were both significantly higher (by $105 \%$ and $43 \%$, respectively) with whey pre-load. Notably, the early insulin response was $96 \%$ higher after whey. Similarly, both total GLP-1 (tGLP-1) and intact GLP-1 (iGLP-1) levels were significantly higher (by $141 \%$ and $298 \%$, respectively) with whey pre-load. Dipeptidyl peptidase 4 plasma activity did not display any significant difference after breakfast between the groups.

Conclusions/interpretation In summary, consumption of whey protein shortly before a high-glycaemic-index breakfast increased the early prandial and late insulin secretion, augmented tGLP-1 and iGLP-1 responses and reduced postprandial glycaemia in type 2 diabetic patients. Whey protein may therefore represent a novel approach for enhancing glucoselowering strategies in type 2 diabetes.

Trial registration ClinicalTrials.gov NCT01571622

Funding The Israeli Ministry of Health and Milk Council funded the research.

Keywords Breakfast · Diabetes · GLP-1 · Metabolic syndrome $\cdot$ Whey
Abbreviations
DPP-4 Dipeptidyl peptidase 4
GIP Glucose-dependent insulinotropic polypeptide
GLP-1 Glucagon-like peptide-1
iGLP-1 Intact GLP-1
tGLP-1 Total GLP-1 


\section{Introduction}

One of the earliest changes in the development of type 2 diabetes is the loss of early postprandial insulin response, a change which contributes to postprandial hyperglycaemia. Therefore, targeting the early postprandial insulin response is an important glucose-lowering strategy in treating type 2 diabetes [1]. One such potential approach is to augment the beta cell response to glucose by a primary stimulus, as has been observed after sequential OGTTs and with IVGTTs or hyperglycaemic clamps $[1,2]$. Another approach is to target the incretin hormone glucagon-like peptide-1 (GLP-1), which is an important mediator for the postprandial insulin response [3]. Indeed, it has been shown that incretin-based therapies, such as GLP-1 receptor agonists [3] and inhibitors of GLP-1 inactivating protease, dipeptidyl peptidase 4 (DPP-4) [4], augment the insulin secretory response to meal ingestion by enhancing mainly the early phase of insulin secretion in type 2 diabetes patients. Whether combining these two approaches by using increased GLP-1 secretion as a primary stimulus to a subsequent meal ingestion may further improve insulin secretion is not known.

In this study, we report that such an approach with a primary stimulus is indeed achievable by using whey protein, which accounts for $20 \%$ of whole-milk protein and is known to stimulate both incretin hormone secretion and insulin secretion [5]. The approach was to serve a pre-meal load of whey protein followed by a regular meal, to examine this prestimulus on postprandial GLP-1 and insulin secretion.

\section{Methods}

Study population The study population comprised 15 participants (electronic supplementary material [ESM] Table 1) with type 2 diabetes. Patients taking anorectic drugs, steroids, medications known to affect gastric motility or oral hypoglycaemic agents other than sulfonylurea or metformin were excluded. The study was approved by the Helsinki Committee of the Wolfson Medical Center in Holon, Israel. The study was registered at ClinicalTrials.gov registration No. NCT01571622.

Study design This was a randomised, open label crossover clinical trial (ESM Fig. 1). Participants were randomised using a coin flip. Each participant consumed two test meals separated by a washout period of at least 2 weeks. At the first visit, participants were randomised to consume a pre-meal drink with either $50 \mathrm{~g}$ [920 kJ (220 kcal)] whey protein concentrate (Beit Haemek Advanced Technologies, Beit Haemek, Israel) dissolved in water or water (placebo). A fasting blood sample $(t=-30 \mathrm{~min})$ was taken and the pre-meal drinks were served. Blood samples were taken at $t=0 \mathrm{~min}$ and, immediately after, participants were served a high-glycaemic-index $1,477 \mathrm{~kJ}$ (353 kcal) breakfast. Thereafter, blood samples were drawn at $t=15,30,60,90,120,150,180 \mathrm{~min}$. Glucose was assessed using an analyser, insulin and C-peptide were measured by electrochemiluminescence immunoassay and total GLP-1 (tGLP-1) and intact GLP-1 (iGLP-1) were quantified by ELISA. DPP-4 activity was assessed as previously described [5]. On the second visit, each participant was crossed over to the opposite pre-meal drink.

Power calculation and statistical analyses The primary endpoints of the study were the AUCs for plasma concentrations of glucose, intact GLP-1 and insulin during the $30 \mathrm{~min}$ following meal ingestion. Secondary endpoints were the $30 \mathrm{~min}$ AUC for concentrations of C-peptide, total GLP-1 and DPP-4. Exploratory analysis also included AUCs for other time intervals. In this study, we examined 15 participants, based on the known variation in AUC for intact GLP-1 and insulin and assuming a $30 \%$ difference by the pre-stimulus and desiring to detect this difference with $80 \%$ probability; this resulted in the requirement of 15 participants and to allow for discontinuation 19 participants were recruited. The results are expressed as mean \pm SEM. For time series, a two-way ANOVA was performed and a least-significant difference $t$ test post hoc analysis was used for comparison between the groups at each time point. For the comparison of fasting with postprandial time points within a group, Dunnet's post hoc test was performed. Student's $t$ test was used for comparing AUCs at different time intervals. In addition, a multivariate ANOVA for repeated measurements was performed, assessing between- and within-subject effects for diet and time.

\section{Results}

Glucose, insulin and C-peptide Fasting glucose, insulin and $\mathrm{C}$-peptide levels did not differ significantly between the two test days or the groups (Fig. 1). In the early interval $\mathrm{AUC}_{0-30 \mathrm{~min}}$, plasma glucose excursions were significantly lower in the whey pre-load group (Fig. 1a, Table 1). Similarly, plasma glucose excursions were significantly lower at the late interval $\mathrm{AUC}_{60-120 \mathrm{~min}}$, and during the complete post-meal period (0-180 $\mathrm{min}$ ), after whey pre-load compared with placebo (Fig. 1a, Table 1).

Whey protein significantly increased the postprandial early insulin release. Plasma insulin levels at the first $30 \mathrm{~min}$ were higher and increased more rapidly after whey pre-load (Fig. 1b, Table 1). Over the late interval, $\mathrm{AUC}_{60-120 \mathrm{~min}}$, and during the complete post-meal period (0-180 min), plasma insulin levels were also significantly higher after whey preload than after placebo (Fig. 1b, Table 1). After meal ingestion, C-peptide levels mirrored those of insulin, with a more rapid and higher increase after whey pre-load (Fig. 1c, Table 1). 
Fig. 1 Meal tolerance test of whey or placebo pre-load. Participants consumed a pre-meal $250 \mathrm{ml}$ drink of either $50 \mathrm{~g}$ whey protein concentrate or water (placebo). Blood samples were taken immediately before the preload $(t=-30 \mathrm{~min})$ and every 30 min thereafter. Participants were served a high-glycaemicindex breakfast instantly after the second blood sample $(t=0)$. For every time point, blood samples were analysed for glucose (a), insulin (b), C-peptide (c), tGLP-1 (d), iGLP-1 (e) and DPP-4 activity (h). In addition, the $\mathrm{iGLP} / \mathrm{tGLP}$ ratio (f) and the correlation of insulin to iGLP ( $r=0.18$, placebo; $r=0.52$, whey) (g) were calculated. Values are means \pm SEM, $n=15,{ }^{*} p<0.05$ vs placebo for the same time point. For parts $(\mathbf{a}-\mathbf{f}, \mathbf{h})$ : black circles, placebo; white circles, whey protein. For part (g): grey circles, placebo; white triangles, whey protein a

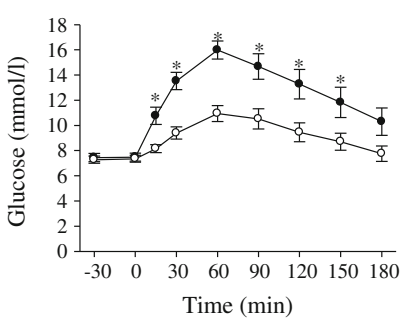

d

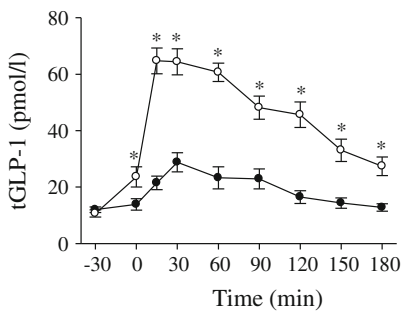

g b

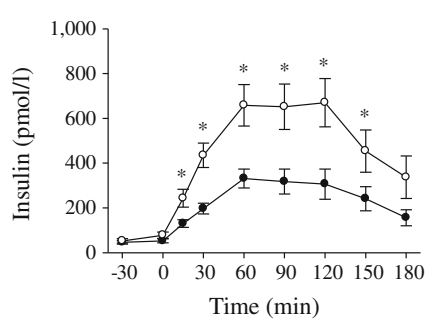

e

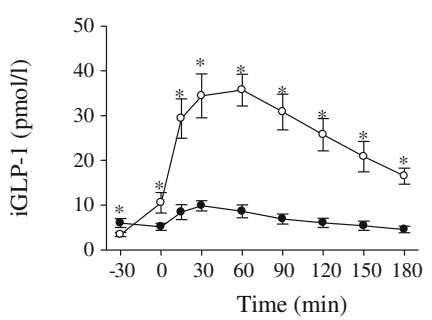

h

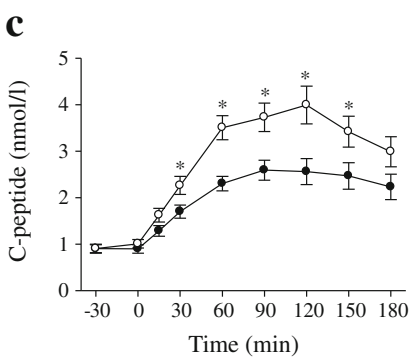

f

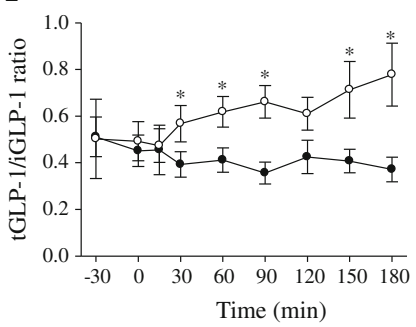

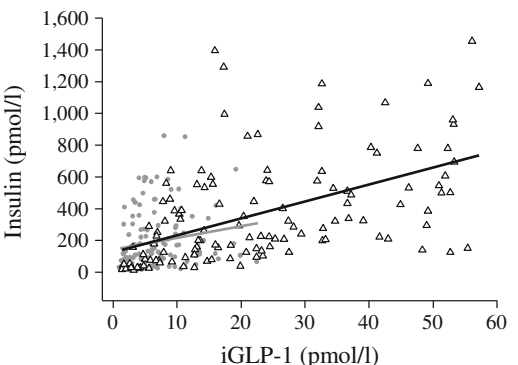

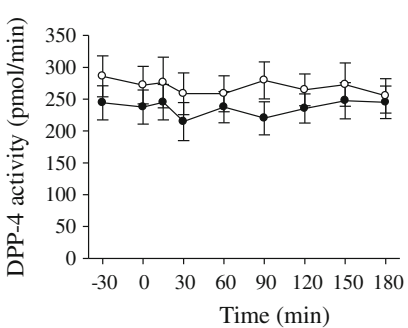

GLP-1 levels and DPP4 activity Higher incremental $\mathrm{AUC}_{\mathrm{iGLP}-1}$ and $\mathrm{AUC}_{\mathrm{tGLP}-1}$ were observed during all intervals after whey pre-load (Table 1). The peak levels of iGLP-1 and tGLP-1 were higher with whey pre-load. Consequently, during the early interval, whey protein pre-load elicited higher $\mathrm{AUC}_{0-30} \mathrm{~min}$ for tGLP-1 and iGLP-1 concentrations (Fig. 1d, e, Table 1). Similarly, during the late interval (60-120 min), increased tGLP-1 and iGLP-1 was observed after whey pre-load. The ratio iGLP-1/tGLP-1 was significantly higher throughout the postprandial period after whey pre-load (Fig. 1f). Positive correlation of insulin to iGLP-1 was marginally significant after placebo, but significant after whey pre-load (Fig. 1g). DPP4 plasma activity did not display any significant difference after breakfast between the placebo and whey pre-loads (Fig. 1h).

\section{Discussion}

In the present study, we show that a breakfast pre-load of whey protein leads to reduced peak and incremental area for post-meal glucose. This glucose-lowering effect of pre- stimulation with whey was associated with rapid and significantly enhanced early and late prandial insulin response. Furthermore, the whey pre-load led to significantly increased postprandial iGLP-1 and tGLP-1 responses, predominantly during the early interval (first $30 \mathrm{~min}$ ). These results are consistent with previous studies indicating that consumption of whey before a meal or with carbohydrates exerts insulinotropic effects and improves post-meal glycaemia in healthy individuals and in those with type 2 diabetes [6]. After the addition of whey, the postprandial glycaemia decreased by $28 \%$, which is a larger reduction than that observed (18\%) $180 \mathrm{~min}$ after different doses of nateglinide, a rapid-acting non-sulfonylurea insulin secretagogue [7], and higher than the reduction (12-24\%) observed after a standardised meal and sulfonylurea therapy (glipizide and glibenclamide [known as glyburide in the USA and Canada]) [8]. The effects of whey preload, nateglinide and sulfonylureas were not compared in a headto-head fashion. The increase in early insulin secretion with whey vs placebo was $\sim 52 \%$, which is comparable with the increase in the early insulin release after breakfast with the addition of $2 \mathrm{mg}$ of repaglinide, a short-acting insulin secretagogue [9]. 
Table 1 AUC for the variables for the different time intervals

\begin{tabular}{|c|c|c|c|c|c|}
\hline Variable & AUC interval (min) ${ }^{\mathrm{a}}$ & Placebo & Whey & $p$ value & Change (\%) \\
\hline \multirow[t]{4}{*}{ Glucose $(\mathrm{mmol} / \mathrm{l} \times \mathrm{min})$} & -30 to 0 & $20.4 \pm 0.9$ & $20 \pm 0.7$ & 0.73 & -2 \\
\hline & $0-30$ & $29 \pm 1.5$ & $22.5 \pm 0.9$ & 0.0008 & -22 \\
\hline & $60-120$ & $79.9 \pm 5.2$ & $56.5 \pm 3.7$ & 0.0011 & -29 \\
\hline & $0-180$ & $213.6 \pm 13.8$ & $154 \pm 8.5$ & 0.001 & -28 \\
\hline \multirow[t]{4}{*}{ Insulin $(\mathrm{pmol} / 1 \times \min )$} & -30 to 0 & $1,472 \pm 241$ & $1,958 \pm 325$ & 0.24 & 33 \\
\hline & $0-30$ & $3,815 \pm 438$ & $7,491 \pm 1,023$ & 0.0026 & 96 \\
\hline & $60-120$ & $19,091 \pm 3,011$ & $39,485 \pm 5,693$ & 0.0037 & 107 \\
\hline & $0-180$ & $44,995 \pm 6,626$ & $92,110 \pm 12,903$ & 0.003 & 105 \\
\hline \multirow[t]{4}{*}{ C-peptide $(\mathrm{nmol} / 1 \times \min )$} & -30 to 0 & $25 \pm 2$ & $27 \pm 3$ & 0.66 & 6 \\
\hline & $0-30$ & $36 \pm 3$ & $45 \pm 4$ & 0.0642 & 26 \\
\hline & $60-120$ & $140 \pm 11$ & $208 \pm 17$ & 0.0025 & 49 \\
\hline & $0-180$ & $367 \pm 30$ & $526 \pm 42$ & 0.0047 & 43 \\
\hline \multirow[t]{4}{*}{$\operatorname{tGLP}(\mathrm{pmol} / 1 \times \min )$} & -30 to 0 & $388 \pm 40$ & $493 \pm 61$ & 0.16 & 27 \\
\hline & $0-30$ & $642 \pm 64$ & $1,631 \pm 114$ & 0.0001 & 154 \\
\hline & $60-120$ & $1,283 \pm 188$ & $3,040 \pm 194$ & 0.0001 & 137 \\
\hline & $0-180$ & $3,576 \pm 416$ & $8,635 \pm 473$ & 0.0001 & 141 \\
\hline \multirow[t]{4}{*}{ iGLP-1 $(\mathrm{pmol} / 1 \times \min )$} & -30 to 0 & $168 \pm 25$ & $209 \pm 34$ & 0.331 & 24 \\
\hline & $0-30$ & $239 \pm 36$ & $812 \pm 107$ & 0.0001 & 240 \\
\hline & $60-120$ & $427 \pm 68$ & $1,845 \pm 203$ & 0.0001 & 332 \\
\hline & $0-180$ & $1,264 \pm 183$ & $5,035 \pm 537$ & 0.0001 & 298 \\
\hline \multirow[t]{4}{*}{ DPP-4 (pmol × min) } & -30 to 0 & $7,230 \pm 782$ & $8,368 \pm 889$ & 0.346 & 16 \\
\hline & $0-30$ & $7,070 \pm 798$ & $8,119 \pm 1,009$ & 0.426 & 15 \\
\hline & $60-120$ & $13,697 \pm 1,458$ & $16,233 \pm 1,597$ & 0.25 & $19 \%$ \\
\hline & $0-180$ & $42,187 \pm 4,416$ & $48,090 \pm 5,100$ & 0.4 & $14 \%$ \\
\hline
\end{tabular}

Data are means \pm SEM, $n=15$

${ }^{\text {a }}$ AUCs of the pre-meal interval ( -30 to $\left.0 \mathrm{~min}\right)$, post-meal intervals $(0-30$ or $60-120 \mathrm{~min})$ and total interval $(0-180 \mathrm{~min})$ were calculated by the trapezoidal rule

An important finding was the more rapid and higher early (30 min) iGLP-1 and tGLP-1 response along with enhanced and prolonged rise of both GLP-1 forms at the late interval (60-120 $\mathrm{min})$ of the postprandial response after the whey protein pre-load. It has been suggested that amino acids and bioactive peptides generated during whey protein gastrointestinal digestion directly stimulate $\mathrm{L}$ cell activity to secrete GLP-1 and other incretin hormones [10]. The response of iGLP-1 after a meal pre-loaded with whey protein has not been explored previously in individuals with type 2 diabetes. The significantly higher ratio between iGLP-1 and tGLP-1 observed after whey protein may reflect reduced degradation of tGLP-1. An obvious suggestion for an explanation of this would be a lower DPP-4 activity after whey protein. We did not, however, detect any differences in DPP-4 plasma activity between placebo and whey groups. This would suggest that altered inactivation of GLP-1 by whey protein is not executed through changes in plasma DPP-4 activity. Instead, whey protein may serve as an endogenous inhibitor of DPP-4 in the gut, attenuating local GLP-1 degradation after its release from the enteroendocrine cells as previously suggested in animal studies [5].

A limitation of the study is that we did not examine the detailed mechanisms involved in the effect of the primary stimulus to enhance GLP-1 and insulin secretion, such as the contribution of changes in gastric emptying, meal absorption or the relative contribution of effects on GLP-1 vs insulin secretion, which need to be examined in further mechanistic studies. However, our findings suggest that whey protein may represent a novel approach for enhancing glucose-lowering strategies in type 2 diabetes.

Funding This study was supported by the Israeli Ministry of Health and Milk Council (grant No. 3-00000-9118).

Duality of interest The authors declare that there is no duality of interest associated with this manuscript.

Contribution statement JW, ZL and DJ designed the study. JW, TG and DJ recruited the participants. DJ, JW, MBa and OF analysed the data. 
BA, DJ and OF wrote the manuscript. YB-D and TG collected the clinical information and analysed the blood samples. BA analysed the DPP4 activity. MBa and MBo performed the statistical analyses. All authors contributed to the acquisition, analysis and interpretation of the data and the drafting and editing of the manuscript. All of the authors approved the final version of the manuscript. DJ is the guarantor of this work.

\section{References}

1. Elahi D (1996) In praise of the hyperglycemic clamp. A method for assessment of beta-cell sensitivity and insulin resistance. Diabetes Care 19:278-286

2. Bonuccelli S, Muscelli E, Gastaldelli A et al (2009) Improved tolerance to sequential glucose loading (Staub-Traugott effect): size and mechanisms. Am J Physiol Endocrinol Metab 297:E532-E537

3. Fehse F, Trautmann M, Holst JJ et al (2005) Exenatide augments first- and second-phase insulin secretion in response to intravenous glucose in subjects with type 2 diabetes. J Clin Endocrinol Metab 90: 5991-5997

4. Balas B, Baig MR, Watson C et al (2007) The dipeptidyl peptidase IV inhibitor vildagliptin suppresses endogenous glucose production and enhances islet function after single-dose administration in type 2 diabetic patients. J Clin Endocrinol Metab 92:1249-1255

5. Gunnarsson PT, Winzell MS, Deacon CF et al (2006) Glucoseinduced incretin hormone release and inactivation are differently modulated by oral fat and protein in mice. Endocrinology 147: 3173-3180

6. Frid AH, Nilsson M, Holst JJ, Bjorck IM (2005) Effect of whey on blood glucose and insulin responses to composite breakfast and lunch meals in type 2 diabetic subjects. Am J Clin Nutr 82:69-75

7. Gribble FM, Manley SE, Levy JC (2001) Randomized dose ranging study of the reduction of fasting and postprandial glucose in type 2 diabetes by nateglinide (A-4166). Diabetes Care 24: $1221-1225$

8. Kitabchi AE, Kaminska E, Fisher JN et al (2000) Comparative efficacy and potency of long-term therapy with glipizide or glyburide in patients with type 2 diabetes mellitus. Am J Med Sci 319: $143-148$

9. Cozma LS, Luzio SD, Dunseath GJ, Langendorg KW, Pieber T, Owens DR (2002) Comparison of the effects of three insulinotropic drugs on plasma insulin levels after a standard meal. Diabetes Care 25:1271-1276

10. Jakubowicz D, Froy O (2013) Biochemical and metabolic mechanisms by which dietary whey protein may combat obesity and type 2 diabetes. J Nutr Biochem 24:1-5 